\title{
Desigualdade Salarial entre Raças no Mercado de Trabalho Urbano Brasileiro: Aspectos Regionais*
}

\author{
Filipe R. Campante ${ }^{* *}$ \\ Anna R. V. Crespo ${ }^{* * *}$ \\ Phillippe G. P. G. Leite ${ }^{* * * *}$
}

Sumário: 1. Introdução; 2. Metodologia e especificação; 3. Discriminação racial de salários no mercado de trabalho brasileiro: perfil nacional; 4. Discriminação racial de salários no mercado de trabalho brasileiro: Sudeste e Nordeste; 5. Conclusão.

Palavras-chave: discriminação racial no Brasil; mercado de trabalho; diferenças regionais no Brasil.

Códigos JEL: J15; J31; J71.

Este artigo insere-se na incipiente literatura sobre discriminação racial no Brasil. De modo análogo à contribuição de Soares (2000), utilizamos a decomposição de Oaxaca-Blinder ao longo da distribuição de salarios na PNAD de 1996 para mensurar o componente de discriminação no diferencial de salários entre brancos e negros, incluindo controles para persistência de desigualdades educacionais. De outra parte, reconhecendo as diferenças econômicas e de composição racial entre regiões no Brasil, estabelecemos um perfil comparativo da discriminação no Nordeste e no Sudeste. Os resultados confirmam o perfil "elitista" da discriminação racial, identificado por Soares, mas esclarecem que parte do componente atribuido à discriminação no mercado de trabalho deve-se à persistência de desigualdades educacionais entre raças. Quanto ao perfil regional, conclui-se que o mercado de trabalho é um locus mais importante do problema racial no Sudeste do que no Nordeste, embora o perfil elitista esteja presente em ambas as regiões.

This paper contributes to the literature on racial discrimination in Brazil. Analogously to Soares (2000), we make use of the OaxacaBlinder decomposition, applied to the whole distribution of wages in the 1996 PNAD, to measure the discrimination component of

*Artigo recebido em abr. 2003 e aprovado em fev. 2004. Os autores agradecem as sugestões e ajuda de Francisco Ferreira e Gustavo Gonzaga, assim como os comentários de Maria Carolina Leme e Bruno Amorin. Todos os erros ficam por nossa responsabilidade.

${ }^{* *}$ Harvard University. E-mail: campante@fas.harvard.edu

*** Princeton University. E-mail: acrespo@princeton.edu

**** Delta/World Bank. E-mail: pleite@worldbank.org 
wage differentials between blacks and whites, controlling for persistent educational inequalities. We also provide a comparative profile of racial discrimination in the Northeast and the Southeast, recognizing the important differences across regions in Brazil, both in terms of economic variables and of racial composition of the population. Our results confirm the "elitist" nature of Brazilian racial discrimination that was identified by Soares, but reveal that part of the component of wage differentials ordinarily attributed to labor market discrimination is actually explained by persistent educational inequalities between races. The regional profile suggests that the labor market is a more important locus of the racial issue in the Southeast than in the Northeast, although the elitist feature is present in both regions.

\section{Introdução}

A discussão a respeito da discriminação racial na literatura econômica brasileira é relativamente incipiente e recente comparada à literatura internacional, conquanto esteja em fase de aceleração. Nesta fase, destacam-se como artigos seminais Henriques (2001), que traça um amplo perfil das desigualdades raciais no Brasil e de sua evolução na década de noventa, Calvalieri e Fernandes (1998) e Soares (2000), que procuram identificar e quantificar a discriminação (de raça e gênero) existente no mercado de trabalho brasileiro. Mais especificamente, este último artigo procura utilizar-se do método de decomposição de Oaxaca - Blinder, olhando para a distribuição como um todo conforme Juhn et alii (1993). A idéia básica do método é servir como ferramenta para separar o que poderia ser atribuído à discriminação no mercado de trabalho - no sentido de remunerações distintas para indivíduos com as mesmas características produtivas em postos de trabalho idênticos - daquilo que estaria associado a diferentes características individuais e/ou de inserção no mercado de trabalho, que poderiam dever-se a outras formas de discriminação externas a este mercado.

A despeito da relevância da contribuição do artigo, algumas considerações podem ser feitas a propósito da metodologia utilizada e dos resultados alcançados. Quanto ao primeiro aspecto, o procedimento por ele adotado consiste em estimar dois modelos distintos, um incluindo controles para nível educacional e idade (como proxy de experiência), e outro adicionando a estes variáveis dummy para regiões, setor industrial, posse de carteira e trabalho por conta própria. Ocorre que, se o segundo modelo está correto, então os coeficientes estimados no primeiro padecem 
de viés de variável omitida, ${ }^{1}$ e a comparação entre os resultados de um e de outro fica comprometida. Desse modo, parece interessante buscar um procedimento que permita separar os diferentes efeitos que compõem o diferencial de remuneração e não se sujeite a esse tipo de observação.

Em segundo lugar, pode-se repensar a própria especificação adotada. De fato, o efeito que se atribui à discriminação é em verdade a soma de todos os efeitos que não são diretamente capturados pelo modelo, por não estarem explicitados na especificação. Sendo assim, é possível que incluir novos controles na estimação do modelo acabe gerando um perfil diferente para a discriminação. Se de outra parte há a óbvia preocupação com a parcimônia do modelo, não parece haver qualquer problema em fazer uso de variáveis que a literatura internacional sobre o tema reputa como relevantes no estudo de diferenciais salariais entre grupos, ainda mais levando em conta que o tamanho das amostras disponíveis para o estudo do tema é mais que suficiente para elidir quaisquer preocupações com relação a graus de liberdade na estimação. Diante disso, é interessante incluir variáveis como educação dos pais (ou especificamente da mãe, de acordo com Ganzach (2000)), que usualmente considera-se como tendo impacto no processo de acumulação de capital humano. Essa variável é particularmente importante para o caso do Brasil, pela forte transmissão intergeracional de educação, conforme destacado em Lam (1999).

Finalmente, porém não menos importante, temos de considerar que o artigo procura retratar a discriminação no mercado de trabalho no âmbito nacional. Contudo, sabemos que o Brasil é um país bastante heterogêneo do ponto de vista regional, não havendo, pois, razão para esperar que o perfil da discriminação no mercado de trabalho seja o mesmo entre as regiões do país. Conhecer em maior detalhe esse perfil regional é sem dúvida relevante, seja com o intuito de obter um retrato mais preciso da questão, seja com a finalidade de desenhar políticas públicas que possam lidar com ela. Este fato é mais discutido em Calvalieri e Fernandes (1998), onde são levadas em consideração as regiões metropolitanas separadamente, encontrando em São Paulo e Salvador, respectivamente, o menor e o maior diferencial de salários. Também Soares (2000) reconhece este aspecto, o que se manifesta na inclusão de variáveis dummy para regiões; no entanto, desse modo só se está levando em conta diferenças entre os interceptos nas regiões, sem enfocar a possibilidade de que os próprios coeficientes, isto é, os "preços" dos diferentes atributos na equação minceriana, variem de uma região para outra.

\footnotetext{
${ }^{1} \mathrm{Na}$ verdade, esta observação e válida, pois acredita-se que as variáveis explicativas omitidas no primeiro modelo são correlacionadas com as demais variáveis independentes. Caso contrário, este problema não seria verificado.
} 
O presente artigo propõe-se a avançar nas três direções mencionadas, atendose, para limitarmos seu escopo, à questão da discriminação racial contra negros no Brasil - sem que isso implique qualquer juízo comparativo entre este tipo particular de discriminação e quaisquer outros. Na primeira direção, concluímos que não há muita diferença entre nossos resultados e os de Soares, tanto do ponto de vista quantitativo como quanto à direção do efeito de cada grupo de características. No que diz respeito à distribuição como um todo, o resultado principal se mantém: a discriminação racial apresenta um caráter "elitista", aumentando à medida que se avança na distribuição de salários. Na segunda direção, mostramos que a inclusão da educação da mãe como variável explicativa adicional reduz o componente de discriminação do diferencial de salários, conforme se verifica na literatura internacional. Nesse sentido, parte do problema racial atribuído ao mercado de trabalho está de fato associado a outras dimensões, como a desigualdade educacional e sua transmissão intergeracional. Finalmente, na terceira linha de análise, nossos resultados revelam diferenças notáveis entre as regiões estudadas separadamente: o mercado de trabalho surge como um locus mais importante da questão racial no Sudeste do que no Nordeste, especialmente quando a educação da mãe é incluída no estudo. O componente "elitista", no entanto, mostra-se robusto à desagregação por regiões.

No intuito de apresentar e discutir tais resultados, o artigo está organizado da seguinte forma: a seção 2 apresenta a metodologia e a especificação adotada, contextualizando-as na literatura sobre o tema; a seção 3 mostra os resultados obtidos para o Brasil, comparando-os com os obtidos por Soares (2000); e a seção 4 introduz os resultados desagregados para as regiões Sudeste e Nordeste, contrastando-os com o perfil nacional. O motivo para nos concentrarmos nestas duas regiões segue de serem elas as mais populosas (cerca de $60 \%$ do total de brancos na amostra, e cerca de $70 \%$ do total de pretos/pardos), e, portanto, permitirem trabalhar com as maiores amostras possíveis de modo a não comprometer a confiabilidade dos resultados, e também do fato de representarem dois extremos em termos de desenvolvimento econômico no país. A seção 5, finalmente, conclui o presente trabalho.

\section{Metodologia e Especificação}

Conforme ressaltado acima, a idéia central deste artigo é tentar determinar o quanto da diferença salarial entre as raças é devido à discriminação no mercado de trabalho. Isto significa que queremos identificar a forma diferenciada com a qual as características dos agentes são "precificadas", apontando quais entre elas têm 
uma maior diferenciação na sua remuneração. Uma vez feito isto, o artigo pretende se voltar para análise regional, destacando o comportamento diferenciado das disparidades salariais entre Nordeste e Sudeste. Com este intuito, a metodologia utilizada não poderia ser outra senão uma adaptação do procedimento de Oaxaca-Blinder. ${ }^{2}$ A idéia básica é estimar separadamente as equações mincerianas para brancos ${ }^{3}$ e negros, incluindo neste último grupo pretos e pardos. Com isto em mãos, podemos transportar os coeficientes estimados em uma regressão para a outra, verificando, assim, a remuneração de um negro caso suas características fossem "precificadas" como as dos brancos. ${ }^{4}$

A diferença entre o que será apresentado aqui e o método de Oaxaca-Blinder tradicional é que, enquanto no último todos os parâmetros estimados eram transportados de uma só vez, neste trabalho as variáveis estão separadas em blocos, de forma que cada conjunto de parâmetros estimados é transportado de cada vez. Isto quer dizer que nós estaremos tentando não só enxergar como um negro seria remunerado caso fosse pago como um branco (salário estimado de um negro usando os parâmetros calculados para os brancos), mas também o quanto cada bloco de parâmetros responde por esta diferença. Assim, as variáveis são segregadas nos seguintes blocos: características individuais, características do mercado de trabalho, características regionais e escolaridade da mãe.

O primeiro bloco, de características individuais, abrange as variáveis (educação, experiência: idade menos idade em que entrou no mercado de trabalho, gênero) que representam aspectos diretamente ligados ao indivíduo em questão. O segundo, de características do mercado de trabalho, junta as variáveis (com ou sem carteira, setor público ou privado, setor de atividade) associadas ao posto de trabalho ocupado pelo indivíduo. O bloco seguinte, de características regionais, representa os controles para a região do país em que o indivíduo trabalha, assim como a situação: área metropolitana e não metropolitana. Finalmente, o último bloco inclui educação da mãe. Um outro motivo para essa divisão é a manutenção de alguma comparabilidade - a despeito de estarmos trabalhando com um ano

\footnotetext{
${ }^{2}$ As referências desta metodologia estão em Oaxaca (1973) e Blinder (1973).

${ }^{3} \mathrm{Na}$ literatura brasileira empírica sobre discriminação encontramos, tradicionalmente, a comparação entre brancos e amarelos versus pretos, pardos e, ocasionalmente, índios. Aqui estamos excluindo da amostra os amarelos e índios por entendermos a inclusão destes grupos como um possivel viés. Cada um deles, respectivamente, tem características sensivelmente diferentes em relação aos grupos onde sao incluídos.

${ }^{4}$ Para atender ao nosso objetivo, as simulações também poderiam ser feitas no sentido contrário, ou seja, verificando o quanto um branco receberia caso fosse remunerado como preto. No entanto, não só por uma questão de interpretação, mas também para a comparabilidade com outros artigos, apenas olhamos as simulações dos salários dos negros.
} 
diferente, e limitando-nos ao Brasil urbano - com os resultados apresentados em Soares (2000): os três primeiros blocos, ressalvadas as modificações na variável escolhida para representar a experiência, representam os aspectos levados em conta naquele artigo, enquanto o quarto acrescenta a variável nova. Desta forma, incorporando os blocos seqüencialmente à simulação, podemos comparar, sujeitos às restrições mencionadas, nossos resultados com os ali obtidos.

Com isso em mente, temos basicamente as seguintes equações mincerianas estimadas para o logaritmo do salário/hora:

$$
\begin{gathered}
y^{B}=\hat{\beta}_{0}^{B}+\hat{\beta}_{1}^{B} X_{1}^{B}+\hat{\beta}_{2}^{B} X_{2}^{B}+\hat{\beta}_{3}^{B} X_{3}^{B}+\hat{\beta}_{4}^{B} X_{4}^{B}+\hat{\epsilon}^{B} \\
y^{N}=\hat{\beta}_{0}^{N}+\hat{\beta}_{1}^{N} X_{1}^{N}+\hat{\beta}_{2}^{N} X_{2}^{N}+\hat{\beta}_{3}^{N} X_{3}^{N}+\hat{\beta}_{4}^{N} X_{4}^{N}+\epsilon^{N}
\end{gathered}
$$

onde os sub-índices dizem respeito aos blocos (o zero é apenas relativo a constante), e os sobre-índices identificam as raças, sendo $B$ indicativo dos brancos e $N$ dos negros. Com estas equações podemos verificar como os negros seriam remunerados caso apenas a constante fosse a mesma dos brancos e em seguida, como ficaria o salário dos negros uma vez que o retorno das características pessoais $\left(X_{1}\right)$ também fossem transportados da primeira para a segunda equação. Continuaríamos nesta seqüência até que todos os coeficientes fossem transportados. Assim, teríamos as seguintes equações:

$$
\begin{gathered}
y_{I}^{N}=\hat{\beta}_{0}^{B}+\hat{\beta}_{1}^{N} X_{1}^{N}+\hat{\beta}_{2}^{N} X_{2}^{N}+\hat{\beta}_{3}^{N} X_{3}^{N}+\hat{\beta}_{4}^{N} X_{4}^{N}+\epsilon^{N} \\
y_{I I}^{N}=\hat{\beta}_{0}^{B}+\hat{\beta}_{1}^{B} X_{1}^{N}+\hat{\beta}_{2}^{N} X_{2}^{N}+\hat{\beta}_{3}^{N} X_{3}^{N}+\hat{\beta}_{4}^{N} X_{4}^{N}+\epsilon^{N} \ldots \\
y_{V I}^{N}=\hat{\beta}_{0}^{B}+\hat{\beta}_{1}^{B} X_{1}^{N}+\hat{\beta}_{2}^{B} X_{2}^{N}+\hat{\beta}_{3}^{B} X_{3}^{N}+\hat{\beta}_{4}^{B} X_{4}^{N}+\epsilon^{N}
\end{gathered}
$$

Ao procurarmos calcular a diferença de salários entre brancos e negros, poderíamos ver a contribuição de cada grupo de parâmetros, separadamente, com as decomposições que se seguem:

$$
y^{B}-y^{N}=\left(y^{B}-y_{K}^{N}\right)+\left(y_{K}^{N}-y^{N}\right)
$$

O primeiro termo do lado direito nos diz o quanto a diferença dos salários aumenta ou diminui em virtude da transferência dos $K$ primeiros blocos de parâmetros, onde $K=1,2,3,4$. Isto nos diz o quanto as características estão respondendo pela desigualdade, uma vez que estão sendo comparados os salários estimados dos dois grupos com os mesmos parâmetros, preservando somente as características. 
Já o segundo termo nos informa sobre o quanto o salário dos negros varia com a inclusão destes parâmetros. Este é denominado o efeito preço, que nos diz o quanto as características dos brancos são remuneradas de forma diferenciada em relação aos negros.

$\mathrm{Na}$ análise regional, feita em seguida, a mesma metodologia é aplicada para os dados do Nordeste e do Sudeste. A única diferença, obviamente, está no bloco de características regionais, que passam a incluir somente controle para a situação geográfica.

Os dados utilizados são da PNAD (Pesquisa Nacional por Amostra de Domicílio) do ano de 1996. A escolha da amostra de 1996 se deve ao fato de existir apenas para este ano o suplemento com informações sobre os pais do chefe de família e do cônjuge. Com isso, a amostra utilizada se limita apenas àqueles que têm como posição na família a categoria de chefe, cônjuge ou filho; todos os demais estão excluídos por falta de informação no que diz respeito aos seus pais, mais especificamente, às suas mães. Também limitamos a amostra apenas a trabalhadores formais e informais, excluindo todos aqueles que se declararam como trabalhadores por conta própria e empregadores. Isto se deve exclusivamente ao argumento de que estamos interessados em traçar um perfil para a discriminação no mercado de trabalho, de forma que só faz sentido nos referirmos àqueles que podem ser discriminados pelos seus empregadores. Além disso, a outra restrição feita à amostra é quanto aos dados dos moradores da área rural, que também foram excluídos. A justificativa está na falta de confiabilidade destes dados, conforme apontado em Ferreira et alii (2000).

Vale notar que, ao estudar o tema discriminação, estamos entrando num assunto controverso: a qualidade da variável utilizada pelo IBGE para classificar cor, a qual utilizamos como sinônimo de "raça". De acordo com o questionário do Censo Demográfico brasileiro, assim como da PNAD, os entrevistados devem se classificar ${ }^{5}$ em uma dentre cinco categorias: branco, preto, pardo, indígena ou amarelo (oriental). Este tipo de classificação nos leva a algumas discussões sobre discriminação e subestimação nos dados relativos aos negros. Em particular, muitas vezes considera-se que no Brasil exista um fenômeno de "embranquecimento" do indivíduo relacionado com a renda, isto é, um indivíduo "pobre" se autoclassificaria como negro ou pardo, enquanto um "não pobre", mas com as mesmas

\footnotetext{
${ }^{5}$ De acordo com artigo publicado no Jornal do Brasil em 1998 por Simon Schwartzman denominado "Cor, Raça, Discriminação e Indentidade Social no Brasil", a opção adotada no passado pelo IBGE (de perguntar pela "cor") foi uma tentativa de encontrar uma forma neutra de se aproximar da questão da raça, sem pretender medi-la diretamente. Os termos propostos - branco, pardo, amarelo, preto - foram pensados como totalmente descritivos, sem nenhuma idéia de que eles servissem como forma de identificação dos grupos.
} 
características, se identificaria como branco. Ademais, no que diz respeito ao objetivo aqui proposto, este fenômeno seria observado mais freqüentemente nas regiões mais desenvolvidas. Reconhecendo a complexidade do tema, o IBGE realizou uma série de encontros com organizações representativas de segmentos da sociedade, além de reuniões com usuários das bases de dados da instituição e especialistas do tema durante os anos de 1997 e 1998, ${ }^{6}$ para avaliar e tentar melhorar a mensuração da variável raça durante a coleta dos dados do Censo em 2000. Durante este período técnicos e pesquisadores analisaram a questão da cor/raça utilisando dados coletados através de formas alternativas de coleta, perguntas abertas, perguntas fechadas e perguntas sobre a origem do entrevistado. Entretanto, nenhum dos trabalhos realizados validava a troca do método-padrão de pergunta fechada. Diante disso, e também em vista do fato de que a questão tampouco é abordada em outros artigos sobre o tema, optamos por nos ater aos termos do IBGE.

\section{Discriminação Racial de Salários no Mercado de Trabalho Bra- sileiro: Perfil Nacional}

\subsection{Perfil da população brasileira}

Desde 1940, a composição demográfica brasileira mudou de um país cuja maioria era branca $(63,47 \%)$ para um país onde apenas cerca de metade da população se declara branca (53,74\%). No período de 1940 a 2000, houve um decréscimo significativo no percentual de pretos, de $14,64 \%$ para $6,21 \%$, enquanto a população parda aumentava consideravelmente. Este fato é intrigante, pois ainda que a taxa média de crescimento populacional aumentasse, ${ }^{7}$ a taxa de crescimento de pardos deveria ser muito maior para explicar este crescimento. Este crescimento do percentual de pardos é costumeiramente associado a uma "desvalorização" dos pretos, que tenderiam a deixar de se classificar como pretos como uma forma de ganhar status na sociedade. Na década de 90, o movimento de valorização da cultura negra parece ter revertido esse processo. Não se pode afirmar com certeza que este movimento mudou os padrões de classificação da população, mas observa-se que a taxa de crescimento de pretos entre 1991 e 2000 foi de 4,17\%, enquanto a de pardos foi de apenas $0,53 \%$. Além disso, pela primeira vez em 60 anos o percentual

\footnotetext{
${ }^{6}$ Diversas propostas foram recebidas para incluir alterações na investigação sobre cor e raça, por parte de pesquisadores e de instituições como Fundação Palmares, Grupo Interministerial de Valorização da População Negra, e outras organizações não-governamentais.

${ }^{7}$ Entre 1950/1960 a taxa de crescimento foi de 2,99\%, entre 1960/1970-2,89\%, entre 1970/1980 - 2,48\%, 1980/1991-1,93\% e 1991-2000 - 1,64\%. Fonte IBGE.
} 
de pretos aumentou entre a população recenseada. O percentual de pretos variou negativamente de 1940 até 1991 quase na mesma intensidade em que o percentual de pardos aumentava, enquanto que em 2000 os sinais se invertem.

Tabela 1

Proporção da População Residente por Cor ou Raça Brasil 1940 - 2000.

\begin{tabular}{l|ccccc}
\hline & \multicolumn{5}{|c}{ Cor ou raça (\%) } \\
Ano & Branco & Preto & Pardo & Amarela & Indígena \\
\hline 1940 & 63,47 & 14,64 & 21,21 & 0,59 & - \\
1950 & 61,66 & 10,96 & 26,54 & 0,63 & - \\
1960 & 61,03 & 8,71 & 29,50 & 0,69 & - \\
1980 & 54,23 & 5,92 & 38,85 & 0,56 & - \\
1991 & 51,56 & 5,00 & 42,45 & 0,43 & 0,20 \\
2000 & 53,74 & 6,21 & 38,45 & 0,45 & 0,43 \\
\hline
\end{tabular}

Com base nos dados coletados pela PNAD em 1996, e agregando os pretos e pardos, as regiões Norte e Nordeste são as que apresentam maior percentual de indivíduos nesta categoria $(72 \% ; 69 \%)$. As regiões Sudeste e Sul são as que apresentam maior percentual de brancos, $66 \%$ e $86 \%$ respectivamente. Em termos absolutos o Nordeste e o Sudeste juntos detêm $73 \%$ da população estimada e apresentam um percentual de pretos/pardos e brancos bem caracterizados, isto é, o Nordeste composto em sua maioria pela população preta/parda e o Sudeste pela população branca.

Tabela 2

Distribuição Percentual da População, por Cor ou Raça, segundo as Grandes Regiões 1996

\begin{tabular}{l|cc|cc}
\hline \multicolumn{1}{c|}{ Grandes Regiões } & \multicolumn{2}{|c|}{ \# em milhões } & \multicolumn{2}{c}{$\%$} \\
\cline { 2 - 5 } Unidades da Federação e & Preta/Parda & Branca & Preta/Parda & Branca \\
Regiões Metropolitanas & & & & \\
\hline Brasil(1) & 68,4 & 85,9 & 44 & 56 \\
Norte(2) & 5,3 & 2,1 & 72 & 28 \\
Nordeste & 31,6 & 14,0 & 69 & 31 \\
Sudeste & 22,8 & 44,4 & 34 & 66 \\
Sul & 3,2 & 20,2 & 14 & 86 \\
Centro-Oeste & 5,4 & 5,1 & 51 & 49 \\
\hline
\end{tabular}

Fonte: Pesquisa nacional por amostra de domicílios 1996.

Tabulação dos autores.

(1) Exclusive a população rural de Rondônia, Acre, Amazonas, Roraima, Pará e Amapá.

(2) Exclusive a população rural.

Complementarmente, ao analisarmos indicadores educacionais e do mercado de trabalho, percebemos perfis bastante distintos entre as raças. Os brancos que 
participam do mercado de trabalho têm em média 2 anos a mais de estudo e conseqüentemente maior rendimento que os pretos/pardos, dada a correlação positiva entre nível educacional e salário. Em média, os brancos do Nordeste têm uma média de anos de estudo $69 \%$ maior do que os pretos/pardos, enquanto que no Sudeste esta taxa cai para $41 \%$. Os trabalhadores brancos da região Nordeste recebem em média rendimentos $95 \%$ superiores ao salário médio dos pretos/pardos (2,98 e 1,53 salários mínimos respectivamente). No Sudeste esta variação sobe para exatos $100 \%$ (5,78 contra 2,89 salários mínimos).

\section{Tabela 3}

População Ocupada, por Cor ou Raça, com Indicação da Média de Anos de Estudo e do Rendimento Médio Mensal em Salários Mínimos e Rendimento Mensal em Reais, segundo as Grandes Regiões - 1996

\begin{tabular}{|c|c|c|c|c|}
\hline \multirow{3}{*}{$\begin{array}{c}\text { Grandes Regiões, } \\
\text { Unidades da } \\
\text { Federação e } \\
\text { Regiões } \\
\text { Metropolitanas }\end{array}$} & \multicolumn{4}{|c|}{ População ocupada, por cor ou raça } \\
\hline & \multicolumn{2}{|r|}{ Branca } & \multicolumn{2}{|c|}{ Preto/Pardo } \\
\hline & $\begin{array}{c}\text { Média de anos } \\
\text { de estudos }\end{array}$ & $\begin{array}{l}\text { Rendimento médio em } \\
\text { salário mínimo }\end{array}$ & $\begin{array}{c}\text { Média de anos } \\
\text { de estudos }\end{array}$ & $\begin{array}{l}\text { Rendimento Médio } \\
\text { em salário mínimo }\end{array}$ \\
\hline Brasil(1) & 6,96 & 4,82 & 4,57 & 2,83 \\
\hline Norte(2) & 7,31 & 4,66 & 5,36 & 2,81 \\
\hline Nordeste & 5,40 & 2,98 & 3,72 & 1,53 \\
\hline Maranhão & 4,85 & 2,94 & 3,06 & 1,08 \\
\hline Piauí & 5,64 & 1,89 & 3,26 & 1,13 \\
\hline Ceará & 5,33 & 2,79 & 3,40 & 1,37 \\
\hline Rio Grande do Norte & 6,30 & 3,13 & 4,22 & 1,77 \\
\hline Paraíba & 4,89 & 2,38 & 3,75 & 1,41 \\
\hline Pernambuco & 6,07 & 3,06 & 4,34 & 1,79 \\
\hline Alagoas & 5,76 & 3.55 & 3,32 & 1,80 \\
\hline Sergipe & 5,18 & 3,75 & 4,41 & 1,98 \\
\hline Bahia & 4,95 & 3,19 & 3,89 & 1,71 \\
\hline Sudeste & 7,60 & 5,78 & 5,39 & 2,89 \\
\hline Minas Gerais & 6,52 & 3,85 & 4,58 & 1,98 \\
\hline Espírito Santo & 6,64 & 3,75 & 5,23 & 2,37 \\
\hline Rio de Janeiro & 8,63 & 6,33 & 6,25 & 3,11 \\
\hline São Paulo & 7,73 & 6,44 & 5,66 & 3,75 \\
\hline Sul & 6,57 & 3,95 & 4,64 & 2,21 \\
\hline Centro-Oeste & 7,04 & 5,08 & 5,20 & 2,89 \\
\hline
\end{tabular}

Fonte: Pesquisa nacional por amostra de domicílios 1996: Tabulação dos autores.

(1) Exclusive a população rural de Rondônia, Acre, Amazonas, Roraima, Pará e Amapá.

(2) Exclusive a população rural.

Ao restringirmos ainda mais a cobertura de análise dos dados, observamos que nas áreas urbanas a diferença entre níveis educacionais e rendimentos médios mantém o mesmo comportamento, mas com valores menos acentuados no Nordeste. A variação de anos de estudo cai de $69 \%$ para $40 \%$ enquanto que a variação de rendimentos diminui de $95 \%$ para $80 \%$. No Sudeste não há muita variação entre os resultados acima e os resultados em áreas urbanas. Isto se deve 
basicamente ao fato de que o Nordeste tem maior área rural do que o Sudeste (37\% contra 11\%). Este diferencial aumenta ainda mais se observarmos apenas o mercado de trabalho urbano, isto é, o mercado de trabalho em áreas rurais no Nordeste detém $41 \%$ dos trabalhadores enquanto que no Sudeste o percentual é de apenas $12,5 \%$.

Os dados apresentados acima são boas ferramentas estatísticas que nos mostram que de fato existe uma diferença entre os indicadores sociais de pretos, pardos e brancos, não importando se olhamos para os níveis educacionais ou para o mercado de trabalho. Porém este tipo de estudo "unidimensional" não nos permite estudar as diversas dimensões de análise de um tema complexo como a discriminação. O modelo apresentado na seção anterior nos permite identificar mais precisamente os determinantes da discriminação no Brasil.

\subsection{Resultados da micro simulação}

Seguindo o procedimento exposto na seção anterior, estimamos o modelo descrito para brancos e negros (por questão de simplificação, a partir deste ponto denominamos negros todos os indivíduos que se classificaram como pretos ou pardos). Os resultados estão expostos na tabela A1, no Apêndice. Em primeiro lugar, analisemos os resultados obtidos quanto às médias das distribuições de salários, antes de considerarmos as distribuições como um todo. Eles estão expostos na tabela 4 .

Tabela 4

Valor Esperado do Logaritmo do Salário/hora, por Raça, e Diferença dos Valores Esperados

\begin{tabular}{ccc}
\hline Branco & Negro & Diferença \\
$E\left(X^{B} \beta^{B}\right)$ & $E\left(X^{N} \beta^{N}\right)$ & $E\left(X^{B} \beta^{B}\right)-E\left(X^{N} \beta^{N}\right)$ \\
\hline 2.17 & 1.64 & 0.53 \\
\hline
\end{tabular}

De início, vemos que a diferença entre as médias dos logaritmos dos salárioshora de negros e brancos é de 0,53 , o que significa que os brancos ganham, em média, cerca de $70 \%$ a mais que os negros. Se colocarmos o intercepto estimado para estes na distribuição de salários daqueles, como apresentado na tabela 5 abaixo, o salário dos negros sobe, porém muito ligeiramente, de modo que podemos concluir que não há grande efeito de discriminação na média: se houver, ele está essencialmente no "preço" dos atributos incluídos na equação minceriana estimada $^{8}$. Uma vez que tenhamos incluído as características individuais, dentre

\footnotetext{
${ }^{8}$ Segundo Jones (1983), é um tanto arbitrária esta conotação dada à diferença nos interceptos,
} 
as quais se destaca a educação, já segue uma diferença expressiva: a educação dos brancos é mais bem remunerada que a dos negros, num montante tal que o termo de discriminação corresponde a $42 \%$ do diferencial de salários. As características do mercado de trabalho reduzem esse termo de discriminação para $39 \%$ do diferencial. A interpretação deste aspecto pode ilustrar a complexidade do tema: observa-se que as características do mercado de trabalho dos negros são melhor remuneradas que a dos brancos, mas isso pode conter de fato algum efeito de discriminação. Grande parte desse efeito, como se depreende da tabela A1, advém da diferença entre os coeficientes da dummy para funcionário público de negros e brancos, ${ }^{9}$ o que quer dizer que vale muito mais para um negro ser funcionário público, em relação a estar no setor privado, do que para um branco. Como no setor público a possibilidade de discriminação é mais restrita, isso pode, de fato, estar indicando um tipo de discriminação presente no setor privado. Somando a isso o fato de que o impacto de não ter carteira é mais severo para o branco do que para o negro (ainda que não muito), podemos inferir que está em operação um mecanismo de discriminação na inserção dos negros no mercado de trabalho. ${ }^{10}$

Adicionando as dummies regionais, o termo de discriminação é reduzido novamente, para 34\%. Comparando com os resultados obtidos por Soares (2000) para $1998,{ }^{11}$ tanto o obtido com a introdução do primeiro bloco como o que segue da introdução dos dois seguintes são consistentes, levando em conta o que aquele autor obtém para homens e mulheres negros. Assim sendo, o uso de uma metodologia mais problemática, quando comparada com a utilizada no presente artigo, não parece distorcer de forma significativa o retrato obtido, uma vez que os efeitos de "qualificação" e "inserção", para usar a nomenclatura empregada naquele artigo, são essencialmente compatíveis.

em virtude do fato de que os mesmos mudam conforme a escolha das dummies omitidas.

${ }^{9}$ Vale notar que o coeficiente para a dummy para funcionário público para brancos é um dos dois únicos em todo o modelo que não são significativos a $5 \%$.

${ }^{10} \mathrm{~A}$ discriminação na inserção não está sendo diretamente considerada neste trabalho. No entanto, artigos recentes, como Juhn (2001), mostram que quando controlada a diferença na inserção no mercado de trabalho, a diferença salarial entre raças é ainda maior.

${ }^{11}$ Levando em consideração que um intervalo de dois anos, sem que tenha havido qualquer marco de ruptura na questão, não parece suficiente para mudanças muito drásticas. 
Tabela 5

Valor Esperado do Logaritmo do Salário dos Negros com Parâmetros Imputados dos Brancos, Efeito Preço e Efeito Característica

\begin{tabular}{|c|c|c|c|}
\hline & $E\left(X^{N} \beta^{B}\right)$ & $\begin{array}{c}\text { Efeito Preço } \\
E\left(X^{N} \beta^{B}\right)-E\left(X^{N} \beta^{N}\right)\end{array}$ & $\begin{array}{c}\text { Efeito Característica } \\
E\left(X^{B} \beta^{B}\right)-E\left(X^{N} \beta^{B}\right)\end{array}$ \\
\hline a. Intercepto & 1.70 & 0.06 & 0.48 \\
\hline b. Características individuais $+a$ & 1.86 & 0.23 & 0.31 \\
\hline $\begin{array}{l}\text { c. Características do mercado } \\
\text { de trabalho }+b\end{array}$ & 1.84 & 0.21 & 0.33 \\
\hline d. Características regionais $+c$ & 1.82 & 0.18 & 0.35 \\
\hline e. Educação da Mãe + $d$ & 1.78 & 0.14 & 0.39 \\
\hline & & \multicolumn{2}{|c|}{$\Delta$} \\
\hline a. Intercepto & 1.70 & $12 \%$ & $89 \%$ \\
\hline b. Características individuais $+a$ & 1.86 & $42 \%$ & $58 \%$ \\
\hline $\begin{array}{l}\text { c. Características do mercado } \\
\text { de trabalho }+b\end{array}$ & 1.84 & $39 \%$ & $61 \%$ \\
\hline d. Características regionais $+c$ & 1.82 & $34 \%$ & $66 \%$ \\
\hline e. Educação da Mãe $+d$ & 1.78 & $27 \%$ & $73 \%$ \\
\hline
\end{tabular}

Vejamos agora, ainda na mesma tabela 5, o que resulta da inclusão da variável de educação da mãe. Nesse caso, conforme esperaríamos tendo por base a literatura internacional (Altonji e Blank (1999:3161)), a educação da mãe tem um impacto de redução no termo de discriminação, ${ }^{12}$ para $27 \%$. Isso sugere que esse tipo de variável pode ajudar a explicar a discriminação racial no Brasil. Voltando a atenção para os coeficientes apresentados na tabela A1, podemos ver que eles são significativos para os níveis mais baixos de escolaridade. Mais ainda, pode-se ver que o impacto é ainda mais negativo para os brancos do que para os negros, o que pode estar relacionado ao fato de que os negros têm em média mães com escolaridade inferior: brancos cujas mães têm pouca escolaridade estão ainda mais distantes da média do que os negros na mesma situação. Note-se ainda que, conforme destacado por Lam (1999), o Brasil apresenta uma forte transmissão intergeracional de educação, de forma que a escolaridade não muda muito entre as gerações para ambas as raças. Ainda assim, a educação da mãe ainda apresenta um impacto adicional, o que mostra que a herança de desigualdade educacional tem um peso considerável sobre a situação de desigualdade racial no Brasil.

\footnotetext{
${ }^{12}$ Ao longo do artigo, as diferenças advindas da disparidade entre os parâmetros serão tratadas como sendo o termo de discriminação. Obviamente a diferença entre os parâmetros não pode ser diretamente interpretada como sendo característico de discriminação, pois existe o efeito de variáveis não observadas, incorporadas no resíduo, que certamente poderiam afetar este termo. Desta forma, como optamos por não transportar a distribuição dos resíduos nas simulações apresentadas, o significado mais apropriado para as diferenças dos coeficientes é, portanto, de limite superior da discriminação - como é feito pelo próprio Oaxaca (1973).
} 


\section{Figura 1}

Diferença em logaritmos do salário/hora entre a distribuição dos negros simulada e a distribuição dos negros original

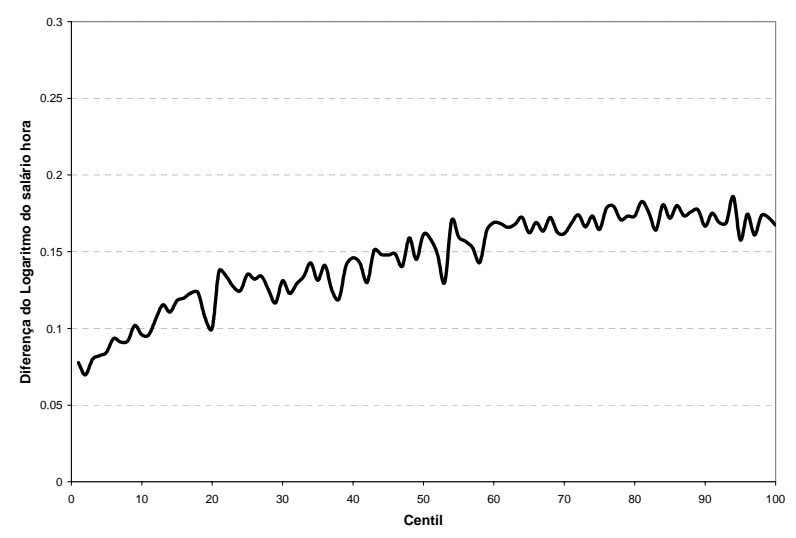

O passo seguinte é passar além da média, investigando como a discriminação se comporta ao longo das distribuições de brancos e negros. Para tanto, podemos recorrer à figura 2 acima, que exibe a log-diferença das Paradas de Pen dos salários-hora de negros na distribuição simulada (com todas as variáveis incluídas) e na distribuição da amostra. Trata-se, então, como em Soares (2000), de uma medida aproximada do ganho percentual que os negros teriam, em cada centil da distribuição, caso fossem remunerados conforme os brancos. Assim, temos uma medida de discriminação ao longo da distribuição. Nota-se que a discriminação segue um padrão crescente ao longo da distribuição, o que confirma o padrão observado por aquele autor (Soares (2000:15)) para os homens negros: a discriminação é mais intensa na cauda superior da distribuição. Reúne-se mais evidência aqui, pois, para a interpretação de que "a sociedade brasileira não aceita que negros ocupem posições favoráveis na estrutura de rendimentos e que quanto mais os negros avançam, mais são discriminados" (Soares (2000:15)). Esse padrão é aqui menos pronunciado do que naquele artigo - variando de $7 \%$ a $17 \%$ de ganho, contra $5 \%$ a $27 \%$-, porém de forma absolutamente consistente, uma vez que ali o padrão é observado apenas para homens.

Em resumo, desta seção parece emergir a conclusão de que alguns dos resultados qualitativos identificados por Soares (2000) perduram, a despeito das modificações metodológicas e de especificação. Mais especificamente, o perfil identificado por aquele autor, segundo o qual a discriminação é maior nos percentis mais altos da distribuição, é corroborado por nossos resultados. Não obstante, 
parte do que foi por ele identificado como discriminação no mercado de trabalho pode ser creditado à herança de desigualdade educacional e seu caráter racial, conforme apreendida pela inclusão da variável de educação da mãe. Isso está de acordo com o observado na literatura internacional sobre o tema. Por fim, a metodologia apresentada aqui nos permitiu diagnosticar um papel importante para a posição de funcionários públicos na determinação do termo de discriminação. Isto quer dizer que, analisando separadamente o mercado de trabalho, podemos perceber que os negros estão em melhores condições sendo funcionários públicos do que os brancos.

\section{Discriminação Racial de Salários no Mercado de Trabalho Bra- sileiro: Sudeste e Nordeste}

Resta ainda averiguar o que resulta de um perfil de discriminação no mercado de trabalho efetuado no plano regional. Consideremos agora o mesmo exercício da seção anterior, agora efetuado separadamente para o Sudeste e o Nordeste. Conforme mencionado anteriormente, e ilustrado pela tabela 6 abaixo, $73 \%$ da população brasileira vive em uma destas duas regiões. Além disso, entre os negros brasileiros, 79\% residem no Nordeste ou no Sudeste. Mais ainda, elas ilustram dois pólos, o Sudeste sendo uma região que concentra $52 \%$ dos brancos brasileiros e o Nordeste que concentra $46 \%$ dos negros.

Tabela 6

Porcentagem da População por Região, por Raça

\begin{tabular}{lcc}
\hline Região & Preto & Branco \\
\hline Nordeste & $46 \%$ & $16 \%$ \\
Sudeste & $33 \%$ & $52 \%$ \\
Outras & $21 \%$ & $32 \%$ \\
Total & $100 \%$ & $100 \%$ \\
\hline
\end{tabular}

Fonte: Pesquisa nacional por amostra de domicílios 1996: Tabulação dos autores.

(1) Exclusive a população rural de Rondônia, Acre, Amazonas, Roraima, Pará e Amapá.

(2) Exclusive a população rural.

Adicionalmente, as duas regiões representam pólos relativamente ao nível de desenvolvimento econômico dentro do Brasil. Isto pode ser visto na tabela 3 (seção 2), que apresenta dados de renda e escolaridade média entre ocupados por Região. As médias do Nordeste são inferiores às do Sudeste, em todos os indicadores. Dados desagregados por Estados também evidenciam esta realidade. 
Passando ao modelo, os resultados da estimação aparecem nas tabelas A2 e A3, no Apêndice. Começando a análise novamente pela média, temos os resultados consolidados na tabela 7. De início, as diferenças salariais entre negros e brancos são bastante parecidas nas duas regiões, (com os brancos recebendo, em média, $61 \%$ e $55 \%$ a mais que os negros no Sudeste e no Nordeste, respectivamente, conforme a tabela 3) o que pode sugerir à primeira vista que o perfil da desigualdade salarial seja homogêneo. No entanto, um olhar mais atento revela notáveis diferenças entre as regiões, que ficam encobertas pelo perfil nacional.

Tabela 7

Valor Esperado do Logaritmo do Salário/hora, por Raça, e Diferença dos Valores Esperados - Nordeste e Sudeste.

\begin{tabular}{ccc}
\hline \multicolumn{2}{c}{ Nordeste } \\
\hline Preto & Branco \\
$E\left(X^{N} \beta^{N}\right)$ & $E\left(X^{B} \beta^{B}\right)$ & $E\left(X^{B} \beta^{B}\right)-E\left(X^{N} \beta^{N}\right)$ \\
\hline 1.37 & 1.82 & 0.44 \\
\hline \multicolumn{3}{c}{ Sudeste } \\
\multicolumn{3}{c}{ Branco } \\
\hline Preto & \multicolumn{2}{c}{0.48} \\
$E\left(X^{N} \beta^{N}\right)$ & $E\left(X^{B} \beta^{B}\right)$ & $E\left(X^{B} \beta^{B}\right)-E\left(X^{N} \beta^{N}\right)$ \\
\hline 1.80 & 2.28 & 0.0
\end{tabular}

Já pela inclusão do intercepto, pode-se perceber um efeito de disparidade na média bem mais pronunciado no Nordeste do que nos resultados nacionais. Nessa região um negro recebe remuneração significativamente menor que a de um branco, já controlada pelas características. A significância desse aspecto pode ser ressaltada se considerarmos que tal intercepto, em nosso modelo, representa a média para um indivíduo na área metropolitana, com carteira, e cuja mãe, bem como ele próprio, possui pós-graduação - vale dizer, com características extremamente favoráveis. Dada a quantidade de negros no Brasil com pós-graduação, um indivíduo como esse está tipicamente num percentil muito superior na distribuição dos negros do que na distribuição dos brancos.

Adicionando as características individuais - destacando-se novamente a educação -, nota-se que a diferenciação na remuneração destas é extremamente expressiva no Sudeste. No Nordeste, ao contrário, o termo de discriminação é reduzido, sugerindo uma "discriminação às avessas", em que a educação dos negros é até melhor remunerada que a dos brancos. Isso pode indicar um efeito de desigualdade educacional entre raças mais pronunciado no Nordeste, uma vez que nessa região o diferencial de características responde pela parte substantiva do diferencial de 
salários. Uma outra abordagem pode sugerir que, como a proporção de negros com dezessete anos ou mais de escolaridade é relativamente muito pequena - o que deve ocorrer principalmente no Nordeste - e que este público possivelmente é mais discriminado do que a média, então o diferencial de salário deles para aqueles com outros níveis de escolaridade deve ser menor do que entre os brancos. Isto levaria a coeficientes mais próximos de zero para as dummies de escolaridade dos negros do que dos brancos, que são menores.

Tabela 8

Valor Esperado do Logaritmo do Salário/hora dos Negros com Parâmetros Imputados dos Brancos, Efeito Preço e Efeito Característica - Nordeste e Sudeste

\begin{tabular}{|c|c|c|c|}
\hline \multicolumn{4}{|c|}{ Região Nordeste } \\
\hline & $E\left(X^{N} \beta^{B}\right)$ & $\begin{array}{c}\text { Efeito Preço } \\
E\left(X^{N} \beta^{B}\right)-E\left(X^{N} \beta^{N}\right)\end{array}$ & $\begin{array}{c}\text { Efeito Característica } \\
E\left(X^{B} \beta^{B}\right)-E\left(X^{N} \beta^{B}\right)\end{array}$ \\
\hline a. Intercepto & 1,65 & 0,17 & 0,27 \\
\hline b. Características individuais + a & 1,55 & 0,27 & 0,17 \\
\hline $\begin{array}{l}\text { c. Características do mercado } \\
\text { de trabalho }+b\end{array}$ & 1,55 & 0,27 & 0,18 \\
\hline d. Características regionais $+\mathrm{c}$ & 1,52 & 0,29 & 0,15 \\
\hline e. Educação da Mãe + d & 1,45 & 0,36 & 0,08 \\
\hline & & \multicolumn{2}{|c|}{$\Delta$} \\
\hline a. Intercepto & 1,65 & $38 \%$ & $62 \%$ \\
\hline b. Características individuais + a & 1,55 & $61 \%$ & $39 \%$ \\
\hline $\begin{array}{l}\text { c. Características do mercado } \\
\text { de trabalho }+b\end{array}$ & 1,55 & $60 \%$ & $40 \%$ \\
\hline d. Características regionais $+\mathrm{c}$ & 1,52 & $66 \%$ & $34 \%$ \\
\hline e. Educação da Mãe + d & 1,45 & $82 \%$ & $18 \%$ \\
\hline \multicolumn{4}{|c|}{ Região Sudeste } \\
\hline \multicolumn{2}{|r|}{$E\left(X^{N} \beta^{B}\right)$} & $\begin{array}{c}\text { Efeito Preço } \\
E\left(X^{N} \beta^{B}\right)-E\left(X^{N} \beta^{N}\right)\end{array}$ & $\begin{array}{c}\text { Efeito Característica } \\
E\left(X^{B} \beta^{B}\right)-E\left(X^{N} \beta^{B}\right)\end{array}$ \\
\hline a. Intercepto & 1,82 & 0,46 & 0,02 \\
\hline b. Características individuais $+\mathrm{a}$ & 2,08 & 0,20 & 0,27 \\
\hline $\begin{array}{l}\text { c. Características do mercado } \\
\text { de trabalho }+b\end{array}$ & 2,05 & 0,24 & 0,24 \\
\hline d. Características regionais $+\mathrm{c}$ & 2,07 & 0,21 & 0,27 \\
\hline \multirow[t]{2}{*}{ e. Educação da Mãe + d } & 2,00 & 0,28 & 0,20 \\
\hline & & \multicolumn{2}{|c|}{$\Delta$} \\
\hline a. Intercepto & 1,82 & $96 \%$ & $4 \%$ \\
\hline b. Características individuais $+\mathrm{a}$ & 2,08 & $43 \%$ & $57 \%$ \\
\hline $\begin{array}{l}\text { c. Características do mercado } \\
\text { de trabalho }+b\end{array}$ & 2,05 & $49 \%$ & $51 \%$ \\
\hline d. Características regionais $+\mathrm{c}$ & 2,07 & $43 \%$ & $57 \%$ \\
\hline e. Educação da Mãe + d & 2,00 & $59 \%$ & $41 \%$ \\
\hline
\end{tabular}

As características do mercado de trabalho, por sua vez, não parecem tão relevantes no Nordeste, mas agem no sentido de aumentar a diferença salarial. No Sudeste, por sua vez, o efeito ocorre na mesma direção do que se verifica no perfil nacional, em magnitude ainda maior. Isso se coaduna com a interpretação 
mencionada anteriormente, relacionada ao papel do emprego no setor público: a proporção de funcionários públicos no Sudeste é mais significativa. Nos coeficientes isso está refletido na maior magnitude assumida no Sudeste, para os negros, em comparação ao Nordeste. De toda forma, o efeito no plano nacional sugere exatamente uma combinação entre os efeitos regionais, o que novamente sugere que aquele está encobrindo um perfil regionalmente heterogêneo. Se levarmos em conta os controles regionais (que no caso limitam-se a diferenciar entre áreas metropolitanas e não-metropolitanas, de modo que a comparação com os resultados nacionais não se dá facilmente), o resultado ocorre na direção exatamente oposta à do efeito anterior, aumentando a disparidade salarial no Sudeste e reduzindo-a no Nordeste: para o negro, no Sudeste, estar numa área não-metropolitana é pior do que para o branco, enquanto o oposto ocorre no Nordeste.

O produto da inclusão desses três blocos de variáveis é exatamente o que temos comparado com a metodologia de Soares (2000). Nesse caso, o que emerge é um perfil bastante distinto entre as duas regiões: de um lado, o Sudeste apresenta um termo de discriminação muito superior à média nacional, enquanto o Nordeste tipifica uma situação de discriminação bem menos significativa. Talvez isso possa ser creditado ao fato de que a proporção de negros na população total da região Nordeste é mais significativa: nessa interpretação, havendo mais negros a discriminação é menos significativa, uma vez que possivelmente o poder de barganha deste grupo é maior em relação ao Sudeste. Portanto, levando em conta apenas os tipos de controle presentes naquele artigo, o perfil de discriminação no mercado de trabalho brasileiro esconde uma situação em que a discriminação é bastante mais severa na região Sudeste. Em suma, há uma grande diferença regional no problema da discriminação: o mercado de trabalho parece ser um locus bem mais fundamental do problema racial no Sudeste do que no Nordeste, ainda que esteja longe de ser desprezivel nesta região.

Tal impressão é confirmada ao incluirmos o quarto bloco, com educação da mãe. Ainda que reduzindo o termo de discriminação no Sudeste de maneira expressiva, analogamente ao que ocorria no perfil nacional, ele ainda é maior nesta região do que no país como um todo. No Nordeste, de outra parte, o termo de discriminação cai praticamente à metade, e torna-se bem menos expressivo. Desse modo, identifica-se uma situação em que a discriminação racial no mercado de trabalho é bem mais importante no Sudeste do que no Nordeste, sendo que nesta região as disparidades salariais são melhor entendidas com referência às desigualdades nas características dos agentes. O perfil nacional, por sua vez, está entre essas duas situações, escondendo a diversidade regional do problema racial.

Resta ainda analisar o que ocorre ao longo das distribuições, da mesma forma 
que na seção precedente. De modo a facilitar a comparação com esta, os resultados da log-diferença entre as Paradas de Pen das duas regiões estão na figura 2 abaixo. Percebe-se claramente que a mensagem aqui é a mesma que a transmitida pelas médias: a discriminação racial de remunerações no mercado de trabalho é inequivocamente maior no Sudeste do que no Nordeste, e o perfil nacional fica exatamente entre as duas. É interessante notar que as curvas não se cruzam em nenhum ponto, o que torna o resultado mais expressivo.

\section{Figura 2}

Diferença em logaritmos do salário/hora entre a distribuição dos negros simulada e a distribuição dos negros original

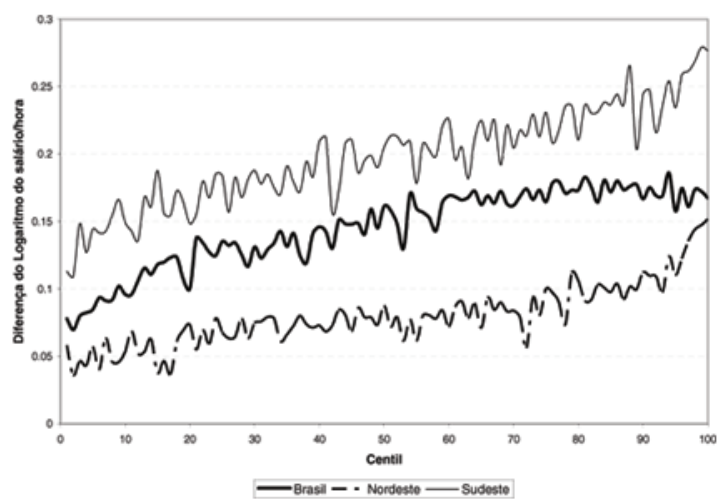

Além disso, ambas as regiões reproduzem de modo bastante nítido o comportamento crescente que foi destacado na análise nacional. Assim sendo, a interpretação a respeito do caráter "elitista" da discriminação racial no Brasil parece se estender para ambos os casos. Aqui, porém, novamente, o Sudeste parece apresentar a questão de forma mais aguda - a variação vai de $11 \%$ a $26 \%$, contra o intervalo de $4 \%$ a $11 \%$ verificado no Nordeste.

De todo modo, perdura a observação de que a discriminação racial no mercado de trabalho é mais significativa no Sudeste do que no Nordeste, diferença encoberta no perfil nacional. Dito de outra forma, nós poderíamos dizer que o mercado de trabalho é um locus mais fundamental para o problema racial no Sudeste do que no Nordeste, no qual as desigualdades educacionais parecem destacar-se mais. 


\section{Conclusão}

O Brasil como um todo apresenta um limite superior para o componente de discriminação da diferença salarial de cerca de $25 \%$ no ano de 1996 , considerandose apenas os trabalhadores com carteira, sem carteira e funcionários públicos. Praticamente todos os resultados encontrados no âmbito nacional são análogos aos apresentados na literatura, principalmente aos artigos citados acima. No entanto, vale destacar que embora um enfoque muito maior seja geralmente dado à questão da discriminação que ocorre dentro do mercado de trabalho, este artigo mostra evidências de que existe também um componente forte da desigualdade advinda da possível discriminação na inserção a este mercado. Conforme destacado acima, um negro está relativamente melhor na posição de funcionário público, e menos prejudicado como sem carteira, em relação as trabalhadores com carteira assinada. Para entrar na primeira posição o critério normalmente utilizado é o de concurso e análise de mérito. Desta forma, uma vez que o indivíduo está capacitado pelas vias legais de atender a um determinado cargo público, a sua raça não será um impeditivo, da mesma forma que o seu salário não deve ser diferenciado por este motivo. Portanto, em relação às outras posições, é muito mais vantajoso para um negro entrar no serviço público, pois neste ele não deve ser discriminado. Contudo, a proporção de negros funcionários públicos é significativamente menor do que a de brancos, esbarrando aqui na questão da desigualdade no acesso à educação. Além disso, sendo eles menos prejudicados como sem carteira, ao invés de com carteira, em relação aos brancos, temos mais evidências de problemas na inserção.

Assim, conforme destacado por Soares (2000), podemos dividir a concretização do salário de um indivíduo em três etapas: formação, inserção e definição salarial. As evidências mostradas acima sugerem que em todas estas etapas os negros estariam sendo prejudicados. Primeiro, porque em média são menos escolarizados, tendo uma formação pior. Em seguida, existem evidências de discriminação na sua inserção. Por fim, suas características são remuneradas de forma diferenciada, como exposto a todo momento neste artigo.

Um ponto fundamental tratado neste trabalho é o antagonismo existente no perfil das diferenças salariais entre as duas regiões: Nordeste e Sudeste, que ficava oculto no tratamento nacional dado ao problema. Foi enfocado aqui que o termo de discriminação é sensivelmente diferente: enquanto na primeira região ele está em torno de 18\%, na segunda ele alcança cerca de 41\%. Isto mostra que no Nordeste a desigualdade salarial é mais bem explicada pelas diferenças nas características dos agentes, e menos é atribuído às diferenciação na "precificação" destas pelo mercado de trabalho. Por outro lado, um perfil oposto é apresentado para o 
Sudeste, onde as diferenças são explicadas de forma significativa pelo termo de discriminação. Com isto em mente, parece difícil que uma política que ignore as diferenças regionais seja traçada pra solucionar o problema, isto é, um plano de ação contra as desigualdades salariais entre raças de não deve ser de caráter nacional.

Obviamente, políticas que focam o problema da desigualdade de educação, principalmente no sentido de tratar a transmissão intergeracional da desigualdade educacional, podem ajudar no combate à disparidade salarial entre as raças em todo o país. Isto porque, não só a educação da mãe tem um peso muito grande no aumento do componente de discriminação no Nordeste, mas, sobretudo, pelo montante pelo qual a educação responde no acesso ao mercado de trabalho, assim como na remuneração nele.

Somando-se a isto a expressiva contribuição do retorno à educação no componente de discriminação no Sudeste, pode-se sugerir que esteja ocorrendo nesta região uma discriminação à la Arrow (1973), com sua teoria de percepção da realidade. Nesta teoria o autor infere que, se o sistema de crenças do empregador diz que trabalhadores negros são menos produtivos do que os brancos, então, este mesmo empregador só contratará negros se o salário deles for menor do que o salário dos brancos. Assim, é como se no Sudeste os empregadores tivessem um sistema de crenças que apontasse a educação dos negros como sendo inferior, de menos qualidade. Isto de fato condiz, em parte, com a realidade - uma vez que os negros têm menos acesso à educação particular, que em média apresenta qualidade muito superior ao ensino público. Portanto, temos um sinal que aponta no sentido de que fundamentalmente a qualidade do ensino oferecida aos negros deveria ser melhorada.

Vistos todos estes pontos, só podemos ter uma conclusão: as políticas de combate à desigualdade podem até, de uma forma geral, ser afins entre as regiões, mas elas devem ser substancialmente diferentes na sua aplicação e na sua forma de focalização. Tratando-se este problema de forma nacional pode-se ajudar a amenizar a desigualdade. Mas a sua solução só pode ser alcançada através de uma análise real das suas causas e conseqüências em cada localidade.

\section{Referências}

Altonji, J. \& Blank, R. (1999). Race and gender in the labor market. In Ashenfelter, O. \& Card, D., editor, The Handbook of Labor Economics. volume 3c.

Arrow, K. (1973). The theory of discrimination. In Ashenfelter, A. \& Rees, A., 
editor, Discrimination in Labor Market, pages 3-33. Princeton University Press, Princeton, N. J.

Blinder, A. S. (1973). Wage discrimination: Reduced form and structural variables. Journal of Human Resources, 8:436-455.

Bourguignon, F., Ferreira, F., \& Leite, P. G. (2002). Beyond Oaxaca-Blinder: Accounting for differences in household income distributions.

Cain, G. G. (1986). The economic analysis of labor market discrimination: A survey. In Ashenfelter, O. A. \& Layard, R., editor, The Handbook of Labor Economics.

Calvalieri, C. \& Fernandes, R. (1998). Diferenciais de salários por gênero e por cor: Uma comparação entre as regiões metropolitanas brasileiras. Revista de Economia Política, 18(1):158-175.

Camargo, J. M., Gonzaga, G. M., Ferreira, F. H. G., Leite, P. G., \& Campante, F. (2001). Segundo relatório de atividades para o ministério do trabalho.

Ferreira, F. H. G., Lanjouw, P., \& Neri, M. (2000). A new poverty profile for Brazil using PPV, PNAD and census data. Texto para Discussão do Departamento de Economia da PUC-Rio, no. 418.

Ganzach, Y. (2000). Parents' education, cognitive ability, educational expectations and educational attainment:interactive effects. British Journal of Educational Psychology, 70:419-441.

Henriques, R. (2001). Desigualdade racial no Brasil: Evolução das condições de vida na década de 90. Texto para Discussão do Ipea, no. 807.

Jones, F. L. (1983). On decomposing the wage gap: A critical comment on Blinder's method. Journal of Human Resorces, 18:126-130.

Juhn, C. (2001). Labor market dropouts and trends in the black white male wages. Working Paper, University of Houston.

Juhn, C., Murphy, K. M., \& Pierce, B. (1993). Wage inequality and the rise in returns to skill. Journal of Political Economy, 101(3):410-442.

Lam, D. (1999). Generating extreme inequality: Schooling, earnings, and intergerational transmission of human capital in South Africa and Brazil. Research Report no. 99-439, University of Michigan. 
Oaxaca, R. L. (1973). Male-female wage differentials in urban labor markets. International Economic Review, 14:693-709.

Roland, E. M. S. (2000). Discriminação racial no mercado de trabalho e o quesito cor. Boletim de Mercado de Trabalho do Ipea, no. 13.

Soares, S. S. D. (2000). O perfil da discriminação no mercado de trabalho - homens negros, mulheres brancas e mulheres negras. Texto para Discussão do Ipea no. 769 . 


\section{Anexo}

Tabela A.1

Brasil - Variável Dependente: Logaritmo do Salário/hora

\begin{tabular}{|c|c|c|c|c|c|c|}
\hline & \multicolumn{3}{|c|}{ Brancos } & \multicolumn{3}{|c|}{ Negros } \\
\hline & Coef. & & $\begin{array}{l}\text { Desvio } \\
\text { Padrão }\end{array}$ & Coef. & & $\begin{array}{l}\text { Desvio } \\
\text { Padrão }\end{array}$ \\
\hline Experiência & 0.045 & * & 0.001 & 0.040 & $*$ & 0.001 \\
\hline Experiência2 & -0.001 & $*$ & 0.000 & -0.001 & $*$ & 0.000 \\
\hline $\begin{array}{l}\text { Dummy se mulher } \\
\text { Anos de estudo }\end{array}$ & -0.276 & $*$ & 0.008 & -0.242 & $*$ & 0.009 \\
\hline 0 & -2.028 & $*$ & 0.044 & -2.094 & $*$ & 0.075 \\
\hline 1 & -1.979 & $*$ & 0.052 & -2.055 & $*$ & 0.078 \\
\hline 2 & -1.901 & $*$ & 0.046 & -2.015 & $*$ & 0.076 \\
\hline 3 & -1.905 & $*$ & 0.044 & -1.954 & $*$ & 0.075 \\
\hline 4 & -1.769 & $*$ & 0.042 & -1.888 & $*$ & 0.074 \\
\hline 5 & -1.775 & $*$ & 0.043 & -1.882 & $*$ & 0.075 \\
\hline 6 & -1.684 & $*$ & 0.043 & -1.751 & $*$ & 0.076 \\
\hline 7 & -1.654 & $*$ & 0.043 & -1.737 & $*$ & 0.076 \\
\hline 8 & -1.502 & $*$ & 0.042 & -1.644 & $*$ & 0.075 \\
\hline 9 & -1.469 & $*$ & 0.045 & -1.615 & $*$ & 0.078 \\
\hline 10 & -1.422 & $*$ & 0.044 & -1.502 & * & 0.077 \\
\hline 11 & -1.106 & $*$ & 0.041 & -1.284 & $*$ & 0.074 \\
\hline 12 & -0.800 & $*$ & 0.050 & -1.005 & $*$ & 0.091 \\
\hline 13 & -0.783 & $*$ & 0.050 & -0.801 & $*$ & 0.092 \\
\hline 14 & -0.609 & $*$ & 0.048 & -0.759 & $*$ & 0.086 \\
\hline 15 & -0.390 & $*$ & 0.042 & -0.567 & $*$ & 0.078 \\
\hline 16 & -0.217 & $*$ & 0.047 & -0.372 & $*$ & 0.092 \\
\hline Anos de estudo da mãe & & & & & & \\
\hline 0 a 4 & -0.179 & $*$ & 0.014 & -0.138 & $*$ & 0.020 \\
\hline 5 a 8 & -0.112 & $*$ & 0.016 & -0.082 & $*$ & 0.023 \\
\hline $\begin{array}{l}\text { Dummy se funcionário } \\
\text { público }\end{array}$ & 0.022 & $* *$ & 0.014 & 0.191 & $*$ & 0.017 \\
\hline $\begin{array}{l}\text { Dummy se sem } \\
\text { carteira }\end{array}$ & -0.237 & * & 0.009 & -0.220 & * & 0.010 \\
\hline Agricultura & -0.276 & $*$ & 0.020 & -0.199 & * & 0.018 \\
\hline Indústria & 0.143 & $*$ & 0.010 & 0.106 & $*$ & 0.012 \\
\hline Construção & 0.073 & $*$ & 0.018 & 0.075 & $*$ & 0.016 \\
\hline Adm Pública & 0.140 & $*$ & 0.016 & 0.091 & $*$ & 0.019 \\
\hline Outros & 0.373 & $*$ & 0.022 & 0.287 & $*$ & 0.040 \\
\hline Norte & -0.147 & $*$ & 0.021 & -0.089 & $*$ & 0.015 \\
\hline Nordeste & -0.403 & $*$ & 0.013 & -0.337 & $*$ & 0.010 \\
\hline Sul & -0.091 & $*$ & 0.008 & -0.039 & * & 0.016 \\
\hline Centro-Oeste & -0.115 & $*$ & 0.013 & 0.002 & 0.013 & \\
\hline Área não metropolitana & -0.198 & $*$ & 0.008 & -0.223 & $*$ & 0.009 \\
\hline Intercepto & 3.486 & $*$ & 0.042 & 3.427 & $*$ & 0.076 \\
\hline $\mathrm{R} 2$ & 0.55 & & 0.46 & & & \\
\hline \# obs. & 36104.00 & & 28624 & & & \\
\hline Root MSE & 0.64 & & 0.6282 & & & \\
\hline
\end{tabular}

* significativo ao nível de significância de $5 \%$.

** significativo ao nível de significância de $10 \%$. 
Tabela A.2

Nordeste - Variável Dependente: Logaritmo do Salário/hora

\begin{tabular}{|c|c|c|c|c|c|c|}
\hline & \multicolumn{3}{|c|}{ Brancos } & \multicolumn{3}{|c|}{ Negros } \\
\hline & \multirow[b]{2}{*}{ Coef. } & \multicolumn{2}{|c|}{ Desvio } & \multirow[b]{2}{*}{ Coef. } & \multirow{2}{*}{\multicolumn{2}{|c|}{$\begin{array}{l}\text { Desvio } \\
\text { Padrão }\end{array}$}} \\
\hline & & & Padrão & & & \\
\hline Experiência & 0.049 & $*$ & 0.005 & 0.044 & $*$ & 0.003 \\
\hline Experiência2 & -0.001 & $*$ & 0.000 & -0.001 & $*$ & 0.000 \\
\hline Dummy se mulher & -0.252 & $*$ & 0.043 & -0.226 & $*$ & 0.027 \\
\hline Anos de estudo & & & & & & \\
\hline 0 & -1.786 & $*$ & 0.214 & -2.297 & $*$ & 0.161 \\
\hline 1 & -1.790 & $*$ & 0.278 & -2.241 & $*$ & 0.176 \\
\hline 2 & -1.598 & $*$ & 0.227 & -2.278 & $*$ & 0.164 \\
\hline 3 & -1.669 & $*$ & 0.223 & -2.193 & $*$ & 0.162 \\
\hline 4 & -1.661 & $*$ & 0.203 & -2.138 & $*$ & 0.161 \\
\hline 5 & -1.435 & $*$ & 0.222 & -2.113 & $*$ & 0.162 \\
\hline 6 & -1.650 & $*$ & 0.215 & -2.016 & $*$ & 0.164 \\
\hline 7 & -1.483 & $*$ & 0.217 & -1.932 & $*$ & 0.165 \\
\hline 8 & -1.423 & $*$ & 0.205 & -1.926 & $*$ & 0.160 \\
\hline 9 & -1.301 & * & 0.221 & -1.753 & $*$ & 0.167 \\
\hline 10 & -1.241 & $*$ & 0.216 & -1.637 & $*$ & 0.169 \\
\hline 11 & -0.933 & $*$ & 0.196 & -1.354 & $*$ & 0.159 \\
\hline 12 & -0.556 & $*$ & 0.227 & -0.850 & $*$ & 0.213 \\
\hline 13 & -0.535 & $*$ & 0.221 & -0.804 & $*$ & 0.232 \\
\hline 14 & -0.622 & $*$ & 0.233 & -0.894 & $*$ & 0.206 \\
\hline 15 & -0.367 & $*$ & 0.203 & -0.564 & $*$ & 0.178 \\
\hline 16 & -0.081 & $*$ & 0.224 & -0.489 & $*$ & 0.195 \\
\hline Anos de estudo da mãe & & & & & & \\
\hline 0 a 4 & -0.244 & $*$ & 0.065 & -0.089 & $*$ & 0.043 \\
\hline 5 a 8 & -0.216 & $*$ & 0.076 & -0.015 & $*$ & 0.054 \\
\hline Dummy se funcionário & & & & & & \\
\hline $\begin{array}{l}\text { público } \\
\text { Dummy se sem }\end{array}$ & 0.196 & $* *$ & 0.058 & 0.134 & $*$ & 0.044 \\
\hline carteira & -0.315 & $*$ & 0.050 & -0.360 & $*$ & 0.029 \\
\hline Agricultura & -0.129 & $*$ & 0.209 & -0.179 & $*$ & 0.055 \\
\hline Indústria & 0.131 & $* * *$ & 0.057 & 0.096 & $*$ & 0.035 \\
\hline Construção & -0.022 & $*$ & 0.093 & 0.080 & $* * *$ & 0.053 \\
\hline Adm Pública & 0.219 & $*$ & 0.066 & 0.151 & $*$ & 0.046 \\
\hline Outros & 0.576 & $*$ & 0.097 & 0.341 & $*$ & 0.136 \\
\hline Área não metropolitana & 0.011 & & 0.048 & 0.087 & $*$ & 0.030 \\
\hline Constante & 2.998 & $*$ & 0.195 & 3.234 & $*$ & 0.160 \\
\hline $\mathrm{R} 2$ & 0.5099 & & 0.5251 & & & \\
\hline \# obs. & 1416 & & 3149 & & & \\
\hline Root MSE & 0.70662 & & 0.64441 & & & \\
\hline
\end{tabular}


Tabela A.3

Sudeste - Variável Dependente: Logaritmo do Salário/hora

\begin{tabular}{|c|c|c|c|c|c|c|}
\hline & \multicolumn{3}{|c|}{ Brancos } & \multicolumn{3}{|c|}{ Negros } \\
\hline & \multirow{2}{*}{\multicolumn{2}{|c|}{ Coef. }} & \multirow{2}{*}{$\begin{array}{l}\text { Desvio } \\
\text { Padrão }\end{array}$} & \multirow[b]{2}{*}{ Coef. } & \multirow{2}{*}{\multicolumn{2}{|c|}{$\begin{array}{l}\text { Desvio } \\
\text { Padrão }\end{array}$}} \\
\hline & & & & & & \\
\hline Experiência & 0.046 & $*$ & 0.001 & 0.039 & $*$ & 0.002 \\
\hline Experiência2 & -0.001 & $*$ & 0.000 & -0.001 & $*$ & 0.000 \\
\hline Dummy se mulher & -0.270 & $*$ & 0.011 & -0.223 & $*$ & 0.015 \\
\hline \multicolumn{7}{|l|}{ Anos de estudo } \\
\hline 0 & -2.053 & $*$ & 0.063 & -2.149 & $*$ & 0.136 \\
\hline 1 & -1.986 & $*$ & 0.077 & -2.167 & $*$ & 0.144 \\
\hline 2 & -1.907 & $*$ & 0.066 & -2.064 & $*$ & 0.137 \\
\hline 3 & -1.914 & $*$ & 0.062 & -2.016 & $*$ & 0.136 \\
\hline 4 & -1.779 & $*$ & 0.059 & -1.967 & $*$ & 0.135 \\
\hline 5 & -1.795 & $*$ & 0.060 & -1.955 & $*$ & 0.136 \\
\hline 6 & -1.710 & $*$ & 0.061 & -1.805 & $*$ & 0.137 \\
\hline 7 & -1.682 & $*$ & 0.061 & -1.804 & $*$ & 0.137 \\
\hline 8 & -1.514 & $*$ & 0.058 & -1.736 & $*$ & 0.135 \\
\hline 9 & -1.490 & $*$ & 0.062 & -1.756 & $*$ & 0.139 \\
\hline 10 & -1.459 & $*$ & 0.062 & -1.624 & $*$ & 0.139 \\
\hline 11 & -1.149 & $*$ & 0.057 & -1.437 & $*$ & 0.135 \\
\hline 12 & -0.798 & $*$ & 0.070 & -1.035 & $*$ & 0.158 \\
\hline 13 & -0.817 & $*$ & 0.072 & -1.078 & $*$ & 0.157 \\
\hline 14 & -0.641 & $*$ & 0.067 & -0.821 & $*$ & 0.153 \\
\hline 15 & -0.397 & $*$ & 0.059 & -0.768 & $*$ & 0.143 \\
\hline 16 & -0.256 & $*$ & 0.067 & -0.719 & $*$ & 0.168 \\
\hline \multicolumn{7}{|l|}{ Anos de estudo da mãe } \\
\hline 0 a 4 & -0.168 & $*$ & 0.020 & -0.086 & $*$ & 0.039 \\
\hline 5 a 8 & -0.101 & $*$ & 0.024 & -0.071 & $* *$ & 0.044 \\
\hline $\begin{array}{l}\text { Dummy se funcionário } \\
\text { público }\end{array}$ & 0.007 & $* *$ & 0.020 & 0.197 & $*$ & 0.030 \\
\hline \multicolumn{7}{|l|}{ Dummy se sem } \\
\hline carteira & -0.221 & $*$ & 0.013 & -0.164 & $*$ & 0.016 \\
\hline Agricultura & -0.239 & $*$ & 0.028 & -0.156 & $*$ & 0.032 \\
\hline Indústria & 0.171 & $*$ & 0.013 & 0.153 & $*$ & 0.018 \\
\hline Construção & 0.076 & $*$ & 0.026 & 0.059 & $*$ & 0.024 \\
\hline Adm Pública & 0.068 & $*$ & 0.023 & 0.035 & $*$ & 0.035 \\
\hline Outros & 0.304 & $*$ & 0.030 & 0.173 & $*$ & 0.061 \\
\hline Área não metropolitana & -0.187 & $*$ & 0.011 & -0.248 & $*$ & 0.014 \\
\hline Constante & 3.475 & $*$ & 0.058 & 3.456 & * & 0.138 \\
\hline $\mathrm{R} 2$ & 0.526 & & 0.369 & & & \\
\hline \# obs. & 16254 & & 9834 & & & \\
\hline Root MSE & 0.642 & & 0.615 & & & \\
\hline
\end{tabular}

